\title{
EXPLORING OH MEGAMASERS
}

\author{
W.A. BAAN \\ Arecibo Observatory \\ Barrio Esperanza, Arecibo, Puerto Rico 00613, USA
}

\begin{abstract}
The $\mathrm{OH}$ megamasers have a distinct parent population of dusty and FIR luminous merger galaxies. Optical, radio and molecular characteristics may be used to further identify the nature of the nuclear activity in the $\mathrm{OH}$ megamaser host galaxies. Recent interferometric data on $\mathrm{OH}$ megamasers Arp 220 and III Zw35 will be used to discuss the spatial distribution of the $\mathrm{OH}$ emission.
\end{abstract}

\section{General Characteristics of Megamasers}

The number of known $\mathrm{OH}$ megamasers has grown to close to 60 sources since 1982 , some of which are still unpublished. About ten extragalactic maser galaxies have been found for each of the $\mathrm{H}_{2} \mathrm{CO}$ and $\mathrm{CH}$ flavors, while there are currently about $20 \mathrm{H}_{2} \mathrm{O}$ sources. The relatively low occurrence rates for finding $\mathrm{OH}$ and $\mathrm{H}_{2} \mathrm{O}$ megamasers among selected samples are due in part to the non-isotropic emission pattern resulting from molecular amplification closely along to the plane of a molecular disk. The opening angle of this axi- symmetric emission pattern varies for each molecule and depends on the size of the amplifying section of the disk and the molecular pumping mechanism. In an earlier paper, a comparison of the FIR luminosity function of $\mathrm{OH}$ megamasers and of luminous IRAS galaxies was used to determine the fraction of FIR galaxies that show $\mathrm{OH}$ megamaser activity. If this fraction only relates to the disk geometry, than one may estimate the opening angles of the $\mathrm{OH}$ emission pattern to be as much as $15^{\circ}$ within the disk/torus structure and a few degrees for $\mathrm{H}_{2} \mathrm{O}$ sources (Baan 1997).

The characteristics of $\mathrm{OH}$ megamasers have been reviewed extensively in the literature (Baan 1991, 1997; Henkel, Baan \& Mauersberger 1993).

\section{General Properties of $\mathrm{OH}$ Megamasers}

Hydroxyl and formaldehyde megamasers show a quadratic relation between the molecular line luminosity and the 60 micron FIR luminosity (see Baan 1989). A quadratic relation may be explained with low-gain amplification of background continuum by foreground inverted molecular gas, and because both the background radio continuum and the amplifying optical depth are proportional to the FIR luminosity. There is also evidence that $\mathrm{H}_{2} \mathrm{O}$ shows a similar dependence on the product of the luminosity of the X-ray pump and the radio continuum (Baan 1997, see also Braatz et al. 1997). The FIR radiation field serves as the dominant pump for the $\mathrm{OH}$ molecules, while for $\mathrm{H}_{2} \mathrm{CO}$ the pumping agent is still in question.

With "low-gain amplification" it is generally understood, that the maser emission is unsaturated and that the amplifying optical depths are a few at most, which is much lower than the gains implied for saturated maser emission. Both diffuse and compact molecular regions may contribute to the total maser emission profiles. Naturally the optical depths will depend on the molecular column density and the intensity of the pumping agent. They are likely to be higher for compact regions than for more diffuse regions. Optical depths of a few have indeed been observed for compact extra-galactic $\mathrm{OH}$ and $\mathrm{H}_{2} \mathrm{O}$ maser emission features. As the effective optical depth of the emission components increases with increasing FIR radiation fields, the simple picture of low-gain amplification will likely break down resulting in deviations from the quadratic $\mathrm{L}_{O H}-\mathrm{L}_{F I R}$ relation. Smaller radio covering factors for the amplifying molecular clouds would raise the required optical 
depth for each cloud. However, the observed quadratic relation suggests that the product of the radio and FIR covering factors remains roughly constant. A slope with $\mathrm{n}<2$ could suggest that the amplification process gets less efficient at higher FIR luminosities (see also Kandalian in this issue).

\section{Optical and Radio Classification of $\mathrm{OH}$ Megamasers}

$\mathrm{OH}$ megamasers are a dominant sub-group of the (super-) luminous FIR galaxies, which at the high luminosity end show mostly optical AGN characteristics and are in part inter- acting systems. At lower FIR luminosities the sample is predominantly composed of starburst (SBN) nuclei. Optical classification studies of the $\mathrm{OH}$ megamaser sample support this general trend with 67 percent showing AGN, LINER, or composite AGN/SBN characteristics and the rest showing pure starburst characteristics (Baan, Salzer \& LeWinter 1998). However, a similar classification on the basis of the radio properties suggests that the majority of 74 percent of the same sample show no or only weak AGN characteristics and are like radio SBNs, which is consistent with the results of other radio surveys (Baan, Haschick \& Besenfelder 1998). On the other hand, more than half of the luminous FIR galaxies may have weak VLBI radio components (Lonsdale et al. 1993), while nearly half the (radio loud) Seyfert 2's and a quarter of the Seyfert 1's may also have compact cores (Norris et al. 1990). There is a clear connection between OH megamasers and sources with Seyfert 2, LINER, or intense SBN nuclei. It has been pointed out before, that all these sources are thought to have edge-on dust/molecular torus structures, which is in clear agreement with the interpretation of molecular megamaser action in edge-on disks.

The discrepancy between the optical and radio classifications of megamasers confirms the uniqueness of these sources. Large concentrations of molecular gas and dust and galactic interactions provide unusual conditions in the nuclear region, which may affect the optical emissions and the diagnostic line ratios. For instance, different emissions may originate at different locations and at different obscuration depths within the galaxy. The majority of the sources have edge-on dust lanes, giving high values of $A_{V}$ for emission from the nucleus itself but lower $A_{V}$ for emissions from the outlying regions above and below the plane. The combination of a modified mass function for the nuclear starburst with a higher mass cutoff and of superwinds emanating from the region could somewhat harden the ionizing radiation field and consequently change the optical emission line ratios. Alternatively, a weak AGN will have to be embedded within a (circum-)nuclear starburst region such that the radio and FIR luminosities are dominated by the (more extended) starburst, while the nuclear optical emission is still dominated by the more obscured AGN component.

\section{Molecular Properties}

The mean density of the ISM in the galactic nuclei strongly affects the starformation process and a higher density could result in a shift in its mass function as (possibly) suggested by the radiooptical characteristics. Similarly the high- density components would be (relatively) enhanced in these nuclei and recent searches for line emission from high density tracer molecules show that they are indeed very prominent. The spectra for a number of molecular transitions in megamaser galaxies have been taken with the IRAM $30 \mathrm{~m}$ and $15 \mathrm{~m}$ SEST telescopes. For instance, the comparison of the line strength of the $\mathrm{CO}(1-0)$ transition, which characterizes low density gas with $\left.\mathrm{n}\left(\mathrm{H}_{2}\right)=10^{\prime} 3\right)$ $\mathrm{cm}^{-3}$, and those of the HCN(1-0) or $\mathrm{CS}(3-2)$ transitions, which characterize higher density gas with $\mathrm{n}\left(\mathrm{H}_{2}\right)=10^{4-5} \mathrm{~cm}^{-3}$, would have to be higher for higher nuclear densities and presumably higher FIR luminosity. This effect may indeed be present in the currently available $\mathrm{CO}(1-0) / \mathrm{HCN}(1-0)$ and $\mathrm{CO}(1-0) / \mathrm{CS}(3-2)$ data (Baan et al. 1998). The ratios of various molecular transitions are quite sensitive to the physical environment in the emission regions and may serve as valuable diagnostics of density, temperature, and the effects of shocks.

\section{Structure of Arp 220}

The prototype megamaser galaxy Arp 220 (IC 4553) has very prominent $\mathrm{OH}$ emission and much weaker $\mathrm{H}_{2} \mathrm{CO}$ emission in the central regions of the two merging (radio) nuclei (Baan \& Haschick 1995). Spectral line VLBI studies with the EVN reveal three high brightness OH components within the main emission line: two components at the eastern nucleus that are separated by $30 \mathrm{pc}$ and one 
close to the western nucleus (Diamond et al. 1990). However, they appear not to coincide with the nuclei themselves as predicted for an AGN - nuclear torus configuration (see Lonsdale et al. 1994). Very recent VLBA results reveal a cluster of compact continuum sources at the nuclei of Arp 220, which have the characteristics of radio supernovae (Smith et al. 1997). On the other hand, the $\mathrm{H}_{2} \mathrm{CO}$ emission structure predominantly comes from the western nucleus with only weak emission from a double structure close to the eastern nucleus and from some intervening regions between the two nuclei (Baan \& Haschick 1995). Recent NIR images of Arp 220 made with NICMOS (Scoville et al. 1997) show an emission structure almost identical to that of the $\mathrm{H}_{2} \mathrm{CO}$ emission. This coincidence may suggest that obscuring dust plays havoc in this region and that the formaldehyde pumping could indeed be related to the NIR radiation fields. The three $\mathrm{OH}$ maser features account for about 40 percent of the single dish flux in the most prominent emission feature, which suggests that more diffuse emission coming from the intervening regions contributes significantly to the total emission.

A simple orbital model has been proposed for the dynamics of the two nuclei that uses only the observed velocity characteristics of the $\mathrm{OH}$ and $\mathrm{H}_{2} \mathrm{CO}$ emissions, which have rather small extremes (Baan \& Haschick 1995). A slightly different model based on the $\mathrm{CO}(1-0)$ line emission proposes distinctly different mean velocities of 5300 and $5600 \mathrm{~km} \mathrm{~s}^{-1}$ for the two nuclei (Scoville et al 1996). There is a distinct $\mathrm{CO}$ emission peak at $5375 \mathrm{~km} \mathrm{~s}^{-1}$, which agrees with the main $\mathrm{OH}$ emission peak. Emission lines for various high-density tracer lines in Arp 220 confirm these more extreme velocities of 5280 and $5580 \mathrm{~km} \mathrm{~s}^{-1}$ for the two nuclei (Baan et al. 1998). On the other hand, both nuclei show compact OH emission at closely the same velocity (Diamond et al. 1990), which suggests a somewhat confused velocity field. The discrepancy between the velocity of the main $\mathrm{OH}$ emission feature and the other molecular data results from the fact that Arp 220 displays two distinct pairs of $1667 / 1665 \mathrm{MHz}$ emission lines at 5375 and $5700 \mathrm{~km} \mathrm{~s}^{-1}$, which accounts for the three emission lines observed in the $\mathrm{OH}$ spectrum (Baan \& Haschick 1987). The most prominent (lowest velocity) component in the spectrum is the strong $1667 \mathrm{MHz}$ line from the West nucleus at $5375 \mathrm{~km} \mathrm{~s}^{-1}$ (with some additional emission from the East), while the middle line is a mixture from East and West. Therefore, the VLBI OH features may after all reveal the velocity field of the molecular regions at both nuclei, while any diffuse emissions would reveal the larger scale molecular structures surrounding the nuclei.

\section{Structure of III Zw35}

The OH megamaser III Zw35 has been studied with MERLIN (Montgomery \& Cohen 1992) and VLBA (Trotter et al. 1997) and shows several extended emission regions with a total extent of 50 pc. At high resolution the emission breaks into compact maser features, which together account for about 50 percent of the flux in the double peaked emission feature. The distribution of the $\mathrm{OH}$ emission and of the missing flux suggest that the maser features originate in a disk centered on the nucleus of III Zw35. One could argue that the two main emission regions with the widest $48 \mathrm{pc}$ separation and velocity separation of about $100 \mathrm{~km} \mathrm{~s}^{-1}$ signify the tangential regions of a keplerian torus, where the amplifying gains may be the largest. If this were true, then the central nuclear mass needs to be $1.4 \times 10^{7} \mathrm{M}_{\odot}$. The torus structure seen in $\mathrm{OH}$ megamasers is thus much larger than the nuclear disks seen in $\mathrm{H}_{2} \mathrm{O}$ megamasers, which is also consistent with current FIR pumping models (see Kylafis \& Pavlakis in this issue). The missing flux in both Arp 220 and III Zw35 are likely to come from lower brightness (maser) emission regions distributed along the front side of a dusty torus as found in the lower resolution observations at MERLIN.

\section{Conclusions}

Megamaser searches of galaxies at high radial velocities have in the past been severely limited by receiver capabilities and spectral coverage. Powerful megamasers have now been found to redshifts of about $80.000 \mathrm{~km} \mathrm{~s}^{-1}$, which is similar to those of luminous (thermal) CO sources. The limited extent of these surveys suggests that $\mathrm{OH}$ megamaser activity is indeed rather common at higher redshifts and that sources can be detected quite easily with high sensitivity telescopes. If $\mathrm{OH}$ megamaser activity extends out to the very red objects in the $\mathrm{z}=1-2$ range, then there would be a large population of detectable $\mathrm{OH}$ megamasers. The velocity structure of the currently highest redshift $\mathrm{OH}$ masers suggests that the dynamic structure of such sources may be quite different from those observed at lower redshifts. For instance, the source IRAS $14070+0525$ has a total line width 
of about $2400 \mathrm{~km} \mathrm{~s}^{-1}$ (Baan et al. 1992). This suggests violent starburst or merging activity in the galaxy, although the radio and optical images of this source do not yet reveal any interaction or merger nature for the source.

The quadratic luminosity relation observed for the $\mathrm{OH}$ sources renders $\mathrm{OH}$ megamasers observable to very high redshifts. For current observing systems, a megamaser is required to have $\mathrm{L}_{O H}$ $=10^{3.8} \mathrm{~L}_{(0)}$ to be observable at $\mathrm{z}=2$ and already two nearby masers have been detected with such luminosities. The $\mathrm{OH}$ megamasers in edge-on Seyfert, LINER, or extreme starburst nuclear systems may serve as an important tool for the study dynamical and molecular properties of nuclei at high redshifts.

\section{References}

Baan, W.A. (1991), Active Nuclei and Molecular Megamasers, Skylines, Third Haystack Observatory Conf., eds. A.D. Haschick and P.T. Ho, (P.A.S.P. Conf. Series) 16, 45-55

Baan, W.A. (1997), Megamasers, High Sensitivity Radio Astronomy, eds. R.J. Davis \& N.J. Jackson, (Cambridge: Cambridge University), p. 73-82

Baan, W.A. et al. (1998), in preparation

Baan, W.A. and Haschick, A.D. (1984), The Peculiar Galaxy IC 4553: VLA-A Observations of the OH Megamaser, Ap.J. 279, 541-549

Baan, W.A. and Haschick, A.D. (1995), Nuclear Antics of IC 4553 (Arp220), Ap.J. 454, 745-748

Baan, W.A. et al. (1992), IRAS 14070+0525: An OH Gigamaser at $\mathrm{z}=0.265$, Ap.J. 396, L99-L102

Baan, W.A., Salzer J.J., and LeWinter, R.D. (1998) Optical Classification of Megamaser Galaxies, Ap.J. submitted

Baan, W.A., Haschick, A.D., and Besenfelder, E. (1998), Radio Classification of Megamaser Galaxies, Ap.J. submitted

Braatz, J., Wilson, A.S., and Henkel, C. (1997), A Survey for $\mathrm{H}_{2} \mathrm{O}$ in Active Galactic Nuclei II. A Comparison of Detected and Undetected Galaxies, Ap.J.S. 110, 321-346

Diamond, P., Norris, R. Baan, W.A., and Booth, R. (1990), VLBI Observations of the OH Megamaser in IC 4553 (Arp220), Ap.J. 340, L49-L52

Henkel, C., Baan, W.A., and Mauersberger, R. (1993), Dense molecular gas in galactic nuclei, Astron. Ap. Rev. 3 , 47-90

Lonsdale, C.J., Smith, H.E., and Lonsdale, C.J. (1993), The Starburst- AGN Connection: A Sensitive VLBI Survey of Luminous IRAS Galaxies, Ap.J. 405, L9-L12

Lonsdale, C.J., Diamond, P., Smith, H.E., and Lonsdale, C.J., Compact $\mathrm{OH}$ megamaser and probable quasar activity in the Galaxy Arp 220, (1994), Nature 370, 117-120

Montgomery, A.S. and Cohen, R.J. (1992), A rotating molecular disc in the megamaser III Zw 35, M.N.R.A.S. 254, $23 \mathrm{p}-26 \mathrm{p}$

Norris, R., Allen, D., Sramek, R., Kesteven, M., and Troup, E. (1990), Compact Radio Cores in Extremely Luminous Far-Infrared Galaxies, Ap.J. 359, 291-295

Scoville, N.Z., Yun, M.S., \& Bryant, P. 1997, Arcsecond Imaging of CO Emission in the Nucleus of Arp220, Ap.J 484, 702-719

Scoville, N.Z., et al., NICMOS Imaging Revealing the Nucleus of Arp 220, (1997), Ap.J. in press

Smith, H.A, Lonsdale, C.J., Lonsdale, C.J., \& Diamond, P.J. 1997, Ap.J. submitted

Trotter, A.S., Moran, L.M., Greenhill, L.J., Zheng, X.-W., Gwinn, C.R. (1997), VLBA Imaging of the OH Maser in III Zw $35, A p . J .485$, L79-L82 